\title{
Genome-wide analysis of DNA methylation in hepatoblastoma tissues
}

\author{
XIMAO CUI*, BAIHUI LIU* , SHAN ZHENG, KUIRAN DONG and RUI DONG \\ Department of Pediatric Surgery, Children's Hospital of Fudan University, Shanghai Key Laboratory of Birth Defects \\ and Key Laboratory of Neonatal Diseases, Ministry of Health, Shanghai 201102, P.R. China
}

Received April 3, 2015; Accepted June 2, 2016

DOI: $10.3892 / \mathrm{ol} .2016 .4789$

\begin{abstract}
DNA methylation has a crucial role in cancer biology. In the present study, a genome-wide analysis of DNA methylation in hepatoblastoma (HB) tissues was performed to verify differential methylation levels between $\mathrm{HB}$ and normal tissues. As alpha-fetoprotein (AFP) has a critical role in $\mathrm{HB}$, $A F P$ methylation levels were also detected using pyrosequencing. Normal and HB liver tissue samples (frozen tissue) were obtained from patients with HB. Genome-wide analysis of DNA methylation in these tissues was performed using an Infinium HumanMethylation450 BeadChip, and the results were confirmed with reverse transcription-quantitative polymerase chain reaction. The Infinium HumanMethylation450 BeadChip demonstrated distinctively less methylation in HB tissues than in non-tumor tissues. In addition, methylation enrichment was observed in positions near the transcription start site of $A F P$, which exhibited lower methylation levels in $\mathrm{HB}$ tissues than in non-tumor liver tissues. Lastly, a significant negative correlation was observed between AFP messenger RNA expression and DNA methylation percentage, using linear Pearson's $R$ correlation coefficients. The present results demonstrate differential methylation levels between HB and normal tissues, and imply that aberrant methylation of $A F P$ in $\mathrm{HB}$ could reflect $\mathrm{HB}$ development. Expansion of these findings could provide useful insight into HB biology.
\end{abstract}

Correspondence to: Professor Kuiran Dong or Dr Rui Dong, Department of Pediatric Surgery, Children's Hospital of Fudan University, Shanghai Key Laboratory of Birth Defects and Key Laboratory of Neonatal Diseases, Ministry of Health, 399 Wan Yuan Road, Shanghai 201102, P.R. China

E-mail: kuirand@hotmail.com

E-mail: dongrui_1982@126.com

*Contributed equally

Key words: epigenetics, liver, cancer, pediatrics, AFP

\section{Introduction}

Hepatoblastoma (HB) is an uncommon liver malignancy in infants and children. It accounts for just over $1 \%$ of pediatric cancers (1), but exhibits increasing incidence in North America and Europe (2). This disease is most commonly diagnosed during a child's first 3 years of life (3); only $5 \%$ of new $\mathrm{HB}$ cases are diagnosed in children older than 4 years (4). The disease occurs significantly more frequently in boys than in girls, for reasons that remain to be determined (4).

HB originates from immature liver precursor cells (2). Histologically, HB can be divided into epithelial or mixed epithelial/mesenchymal tissues (5). The majority of HB cases are epithelial in origin, and consist of a mixture of embryonal and fetal cell types (5). Approximately 5\% of HB cases are of the small cell undifferentiated subtype (5). Currently, surgical resection, adjuvant chemotherapy and liver transplantation are the only options to treat HB (2). Thus, novel strategies are required to improve the current understanding of HB biology and to provide targets for therapy or early detection of the disease.

Methylation of DNA is the only known genetically programmed DNA modification process in mammals; it regulates several biological processes, including gene transcription, $\mathrm{X}$ chromosome inactivation, genomic imprinting and chromatin modification (6-8). Methylation changes in $\mathrm{CpG}$ islands (CGIs) and $\mathrm{CpG}$ shores (low $\mathrm{CpG}$ density areas within $2 \mathrm{~kb}$ of CGIs), as well as $\mathrm{CpG}$ shelves (low $\mathrm{CpG}$ density areas within 2-4 kb of CGIs) (9), affect gene expression. Hypomethylation of promoter regions of crucial genes can activate relevant gene expression and may contribute to tumorigenesis (10-12).

Several genome-wide analyses had been conducted in different types of tumors. For instance, Revill et al revealed sphingomyelin phosphodiesterase 3 as a potent tumor suppressor gene, which is hypermethylated in primary hepatocellular carcinoma (13). The present study aimed to determine whether changes in methylation status could affect HB by analyzing HB tissue samples and paired distant noncancerous tissues to profile differentially methylated genes in the disease.

\section{Materials and methods}

$H B$ specimens and clinical information. Matched pairs of $\mathrm{HB}$ and adjacent non-tumor tissues were obtained from the 
surgical specimen archives of the Children's Hospital of Fudan University (Shanghai, China) from 10 patients who underwent partial hepatectomy, and none of them had received radiation therapy or chemotherapy prior to tumour biopsy or resection; their tissue pathology results confirmed $\mathrm{HB}$ with $>80 \%$ viable tumor cells. The patients did not undergo any prior chemotherapy or other forms of therapy, and underwent standardized treatment post-operation. Clinical data were obtained retrospectively from clinical files (Table I). In total, 6 patients were of the mixed embryonal/fetal subtype, and the remaining 4 patients were of the epithelial subtype. The parents of the patients from whom these samples were obtained provided their written informed consent to participate in the study, and the Ethics Committee of the Children's Hospital of Fudan University approved the study as well as the consent procedure.

Genomic DNA was extracted from $10 \mathrm{HB}$ primary tumors (fresh frozen tissue) and adjacent non-tumor tissues, of which, 3 were subjected to Infinium HumanMethylation450 BeadChip analysis (Table I). Total RNA was extracted from 10 matched HB tumor and non-tumor pairs, reverse transcribed to complementary DNA (cDNA) and then subjected to quantitative polymerase chain reaction (qPCR) analysis of alpha-fetoprotein (AFP) messenger RNA (mRNA) expression.

Infinium HumanMethylation450 BeadChip. Samples from 3 patients were analyzed with Infinium HumanMethylation450 BeadChip (Illumina Inc., San Diego, CA, USA), which is a cost-effective approach to quickly analyze each individual's methylome (14). The DNA was treated with bisulfite and hybridized to arrays according to the manufacturer's protocol. Raw data were adjusted for dye bias and quantile-normalized with data derived from Infinium I or Infinium II assay chemistry probes considered separately (Illumina Inc., San Diego, CA, USA). This high-throughput platform enabled quantitative evaluation of methylation levels with single-nucleotide resolution. The $\beta$-values were used to reflect methylation status, ranging from 0 to 1 , where 0 is unmethylated and 1 is fully methylated.

Gene function analysis. The predicted target genes were input into the Database for Annotation, Visualization and Integrated Discovery (http://david.abcc.ncifcrf.gov/), which uses Gene Ontology (GO) to identify the molecular function of the profiled genes (15). The Kyoto Encyclopedia of Genes and Genomes (KEGG) database (http://www.genome.ad.jp/kegg/) was used to analyze the potential functions of these target genes in the pathways. $\mathrm{P}<0.05$ was used as the cut-off value.

Reverse transcription (RT)-qPCR. Total cellular RNA was isolated from HB and normal tissues using TRIzol reagent (Invitrogen; Thermo Fisher Scientific, Inc., Waltham, MA, USA) and then reverse transcribed using a PrimeScript RT reagent kit (Perfect Real Time) with gDNA Eraser (Takara Biotechnology Co., Ltd., Dalian, China) following the manufacturer's protocol. The expression of $A F P$ was analyzed by qPCR with a SYBRGreen PCR kit (Takara Biotechnology Co., Ltd.). The AFP primers used for q-PCR were: Forward, 5'-GTGGTCAGTTTGCAGCATTC-3' and reverse, 5'-AGA GGAGATGTGCTGGATTG-3' (length, 110 bp). Glyceraldehyde 3-phosphate dehydrogenase mRNA was used as the

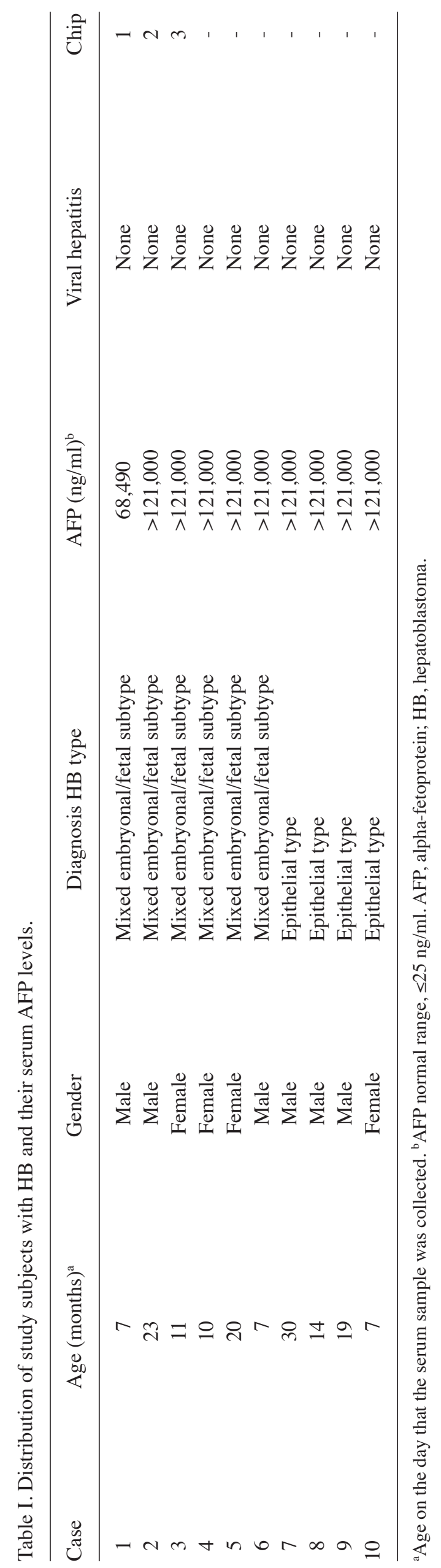




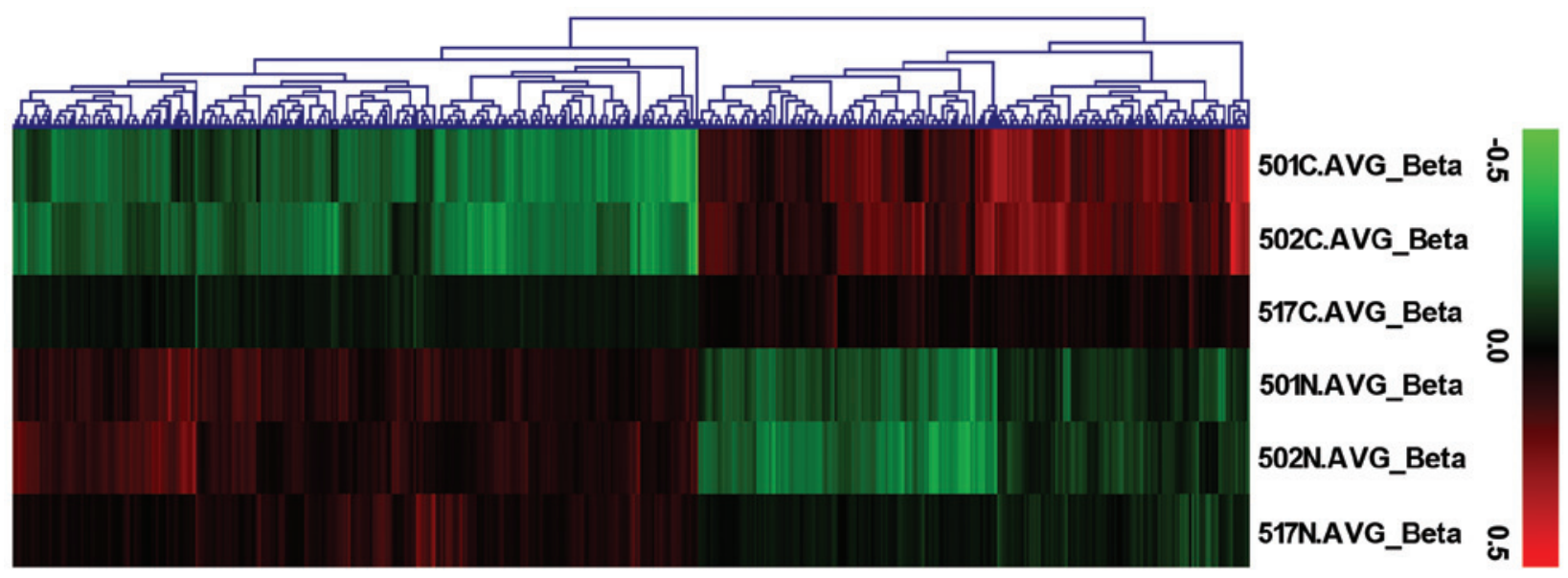

Figure 1. Infinium HumanMethylation450 BeadChip analysis revealed an unsupervised hierarchical clustering of samples based on their $\beta$-values from the $\mathrm{CpG}$ loci. Rows represent the samples (three cancer tissues and three normal tissues) and columns represent CpGs. Green $\beta$-values indicate hypomethylation and red hypermethylation. C.AVG_Beta, cancer tissue denomination; N.AVG_Beta, non-cancer/normal tissue denomination.

internal control, with forward 5'-AAAGCCCACTCCAGC ATC-3' and reverse 5'-TAGCGAGCAGCCCAAAGA-3 primers. The PCR cycling conditions were as follows: $95^{\circ} \mathrm{C}$ for $15 \mathrm{sec}$, followed by $95^{\circ} \mathrm{C}$ for $5 \mathrm{sec}$ and $60^{\circ} \mathrm{C}$ for $31 \mathrm{sec}$, for 40 cycles. For quantitative results, $A F P$ expression was represented as fold-change by the $2^{-\Delta \Delta \mathrm{Cq}}$ method and statistically analyzed (16).

Statistical analyses. Statistical analyses and graphical depiction of data were conducted with GraphPad Prism 5.0 (GraphPad Software, Inc., La Jolla, CA, USA). Results were presented as the mean \pm standard error of the mean and were evaluated with Student's $t$-test (two-tailed) unless otherwise specified (paired $t$-test or Pearson's correlation). Certain statistical calculations were performed using SPSS version 19.0 (IBM SPSS, Armonk, NY, USA). P $<0.05$ was considered to indicate a statistically significant difference.

\section{Results}

Hierarchical clustering analysis by Infinium HumanMethylation450 BeadChip and functional annotations of differentially methylated $C p G$ sites. The Infinium HumanMethylation450 BeadChip was used to obtain data from an independent cohort of $3 \mathrm{HB}$-non-tumor tissue pairs (probe call rate, $>99 \%$ for all samples). Principal component analysis revealed separation of HB-non-tumor pairs and demonstrated the methylation levels of HB tissues to be significantly lower than those of non-tumor tissues (Fig. 1).

Next, GO and KEGG pathway database categories were used to analyze the 524 differential methylated genes identified. Cell adhesion, blood coagulation and nervous system development were observed to be the three most affected biological processes; while cytoplasm, plasma membrane and integral plasma membrane structures were observed to be the three most affected cellular components; and protein binding, nucleotide binding and adenosine triphosphate binding were observed to be the three most affected molecular functions (Fig. 2A-C). Pathway-based analyses revealed significant enrichment for genes in cancer pathways (Fig. 2D).
Aberrant methylation of AFP in $H B$. The methylation microarray indicated enriched methylation of positions near the transcription start site of AFP (Fig. 3). A representative illustration of $A F P$ methylation levels in a matched HB-non-tumor tissue pair is shown in Fig. 4. As HB tissues had lower AFP methylation levels than non-tumor liver tissues, aberrant methylation may be a tumor-specific event in HB.

Correlation of AFP $m R N A$ expression and percentage of $D N A$ methylation. The expression levels of the AFP gene in $10 \mathrm{HB}$-non-tumor liver tissue pairs were observed to be significantly higher in the HB tissues than in their non-tumor counterparts (Fig. 5A). The correlations between AFP mRNA expression and DNA methylation percentage were tested using the linear Pearson's $R$ correlation coefficients, which were interpreted using the scale provided by Salkin $(R=1-0.8$, very strong; $R=0.8-0.6$, strong; $R=0.6-0.4$, moderate; $R=0.4-0.2$, weak; and $R=0.2-0.0$, very weak or not correlated) (17) (Fig. 5B), and were observed to be negatively correlated $(R=0.89 ; \mathrm{P}<0.05)$.

\section{Discussion}

Although HB is an uncommon cancer in the general population, it is the most common liver tumor in children (4). Its etiology is still unknown, but it has been associated with familial adenomatous polyposis (18), low and high birth weights (19) and constitutional trisomy 18 (20). Mutations in numerous components of the wingless-related integration site/ $\beta$-catenin signaling pathway are also often present in HB tissues (21).

Although the mechanisms responsible for $\mathrm{HB}$ development have been extensively studied for the last two decades, the pathogenesis of this disease is still vague, and the majority of its altered gene expression and regulation remain to be delineated. The present authors recently sequenced the HB exome and identified a novel oncogene (caprin family member 2) and three tumor suppressors (speckle-type POZ protein, olfactory receptor family 5 subfamily I member 1 and cell division cycle 20B) that influence HB cell growth (22). A link between long non-coding RNA and HB was also detected (23). 
A

GO biological process

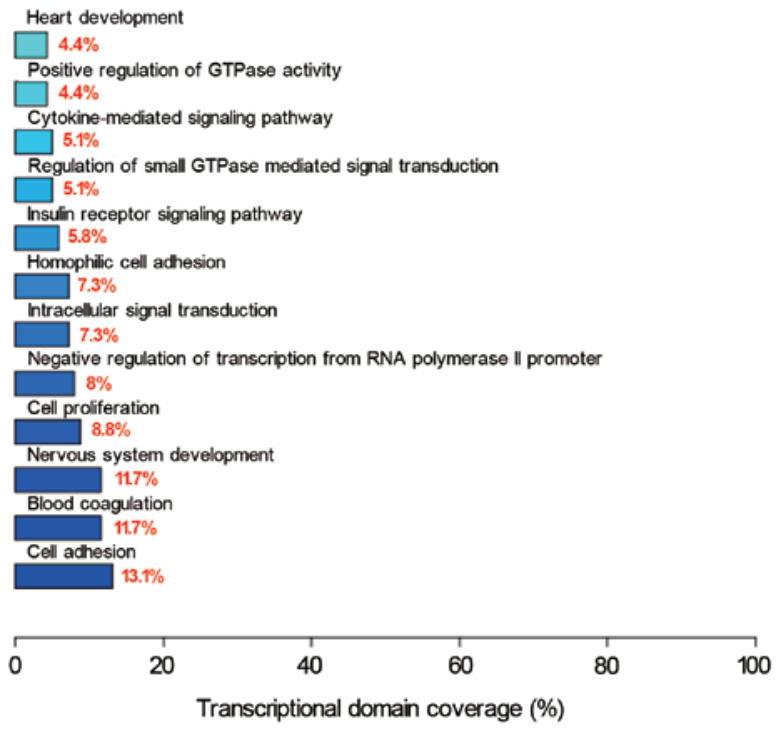

C

\section{GO molecular function}

\begin{tabular}{l} 
Structural constituent of muscle \\
$18 \%$ \\
cAMP binding \\
$1.8 \%$ \\
cAMP-dependent protein kinase regulator activity \\
$1.8 \%$ \\
lonotropic glutamate receptor binding \\
$1.8 \%$ \\
Insulin receptor binding \\
$2.4 \%$ \\
Protein tyrosine kinase activity \\
$3 \%$ \\
SH3 domain binding \\
$4.1 \%$ \\
Guanyl-nucleotide exchange factor activity \\
$4.1 \%$ \\
Identical protein binding \\
7 ATP binding $21.9 \%$ \\
Nucleotide binding \\
Protein binding $29 \%$ \\
\hline
\end{tabular}

\begin{tabular}{rrrrrr}
\hline 0 & 20 & 40 & 60 & 80 & 100
\end{tabular}

Transcriptional domain coverage (\%)
B

\section{GO cellular component}

CAMP-dependent protein kinase complex

| $1.6 \%$

Basement membrane

$2.7 \%$

Postsynaptic density

$27 \%$

Caveola

$2.7 \%$

$\square^{4.4 \%}$

Neuronal cell body

$4.4 \%$

Cell surface

$4.9 \%$

Microsom

$5.5 \%$

Cell junction

$8.8 \%$

Integral to plasma membrane

$17 \%$

Plasma membrane

Plasma membrane $43.4 \%$

Cytoplasm

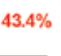

$\begin{array}{llllll}0 & 20 & 40 & 60 & 80 & 100\end{array}$

Transcriptional domain coverage (\%)

D

\section{KEGG}

Pantothenate and COA biosynthesis

$\square .2 \%$

Phenylalanine metabolsm

$6.2 \%$

Cell adhesion molecules

$18.8 \%$

Adherens junction

18.8\%

Leukocyte transendothelial migration

$18.8 \%$

Endocytosis

$31.2 \%$

Pathways in cancer

$37.5 \%$

\begin{tabular}{llllll}
\hline & 20 & 40 & 60 & 80 & 100
\end{tabular}

Transcriptional domain coverage (\%)

Figure 2. GO enrichment and KEGG pathway-based analyses of differentially methylated sites-targets. GO analysis of differentially methylated sites-target genes according to (A) biological process, (B) cellular component and (C) molecular function. (D) KEGG pathway-based analysis of differentially methylated sites-target genes. GO, Gene Ontology; KEGG, Kyoto Encyclopedia of Genes and Genomes; CoA, coenzyme A; GTP, guanosine-5'-triphosphate; cAMP, cyclic adenosine 3',5'-monophosphate; ATP, adenosine triphosphate; SH3, SRC homology 3.

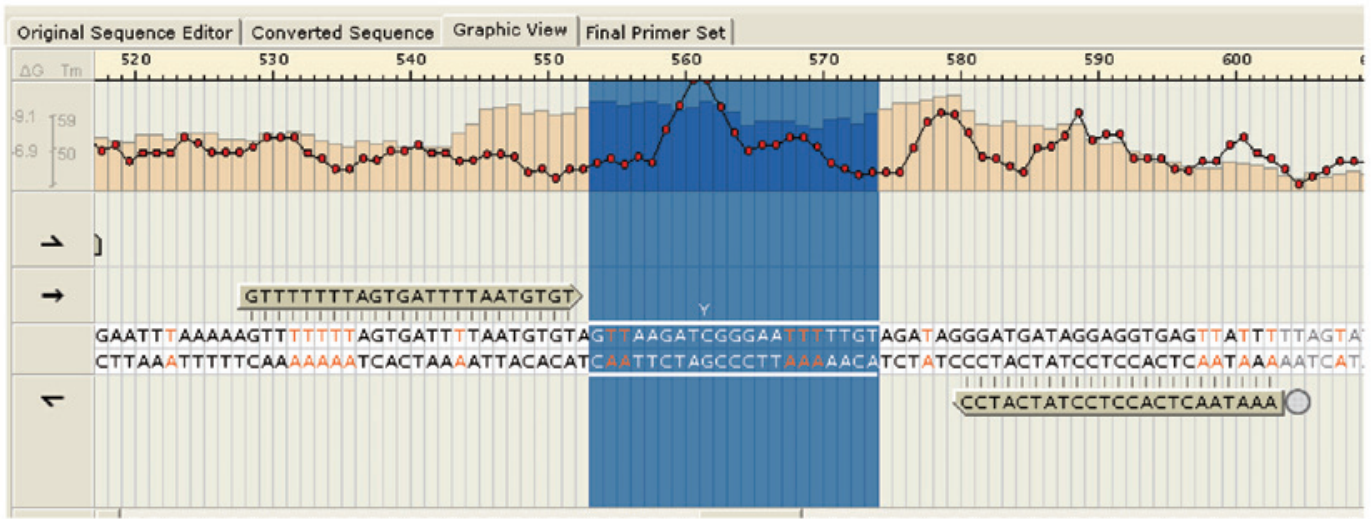

Figure 3. Methylation position enrichment near the transcription start site of alpha-fetoprotein [cg10778295 (TSS1500)] revealed hypomethylated regions. Tm, melting temperature. 
A

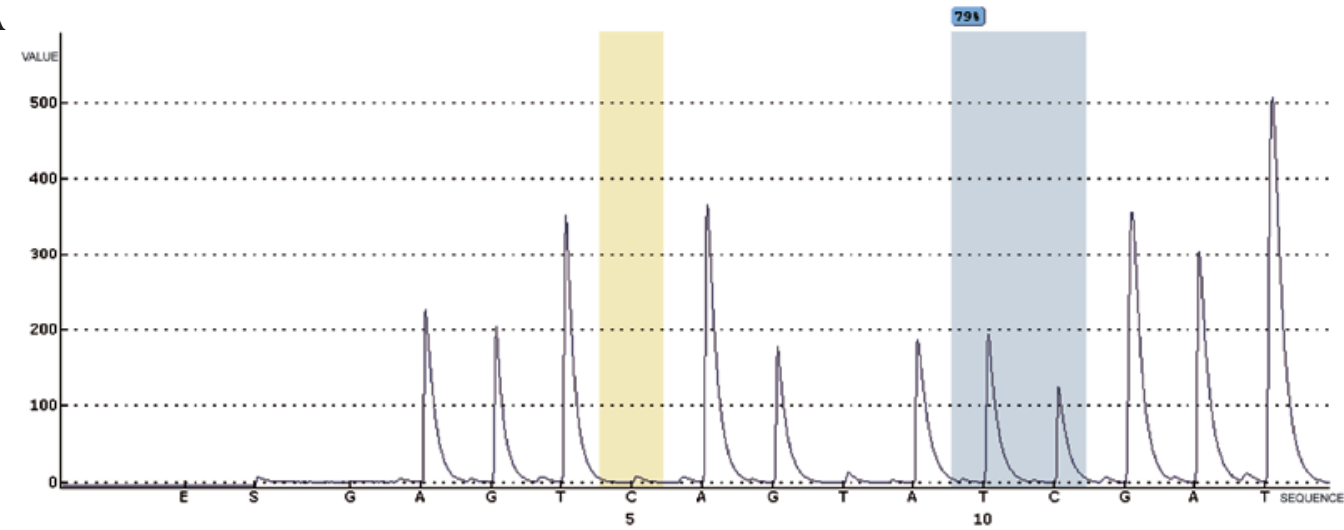

B

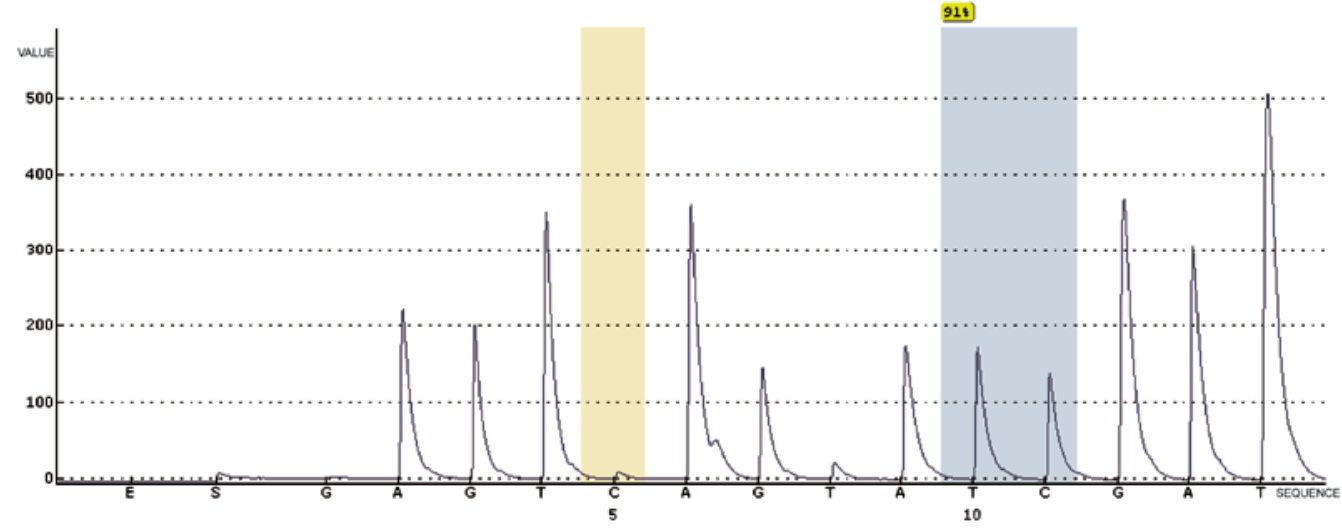

Figure 4. Representative measurements of $A F P$ methylation using pyrosequencing. (A) AFP locus cg10778295 methylation status in cancer tissues (79\% methylation). (B) AFP locus cg10778295 methylation status in normal tissues (91\% methylation). The methylation percentages are percentages of $\mathrm{C}$ at each $\mathrm{CpG}$ site after bisulfite conversion. Orange bar indicates no residual $\mathrm{C}$ at the non- $\mathrm{CpG}$ site, ensuring complete bisulfite conversion. AFP, alpha-fetoprotein .

A

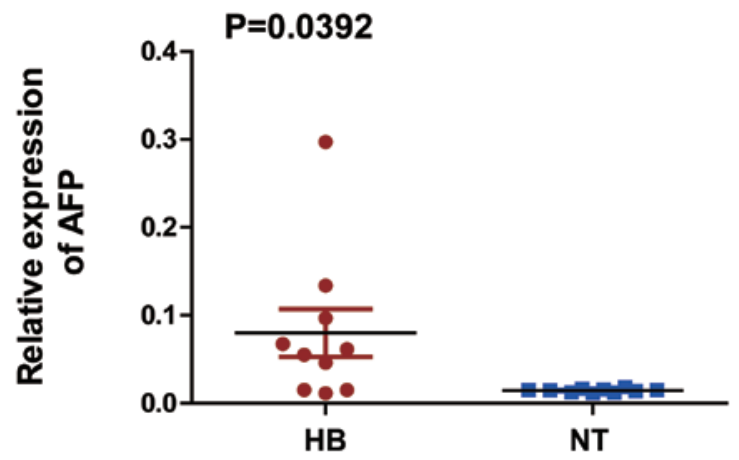

B

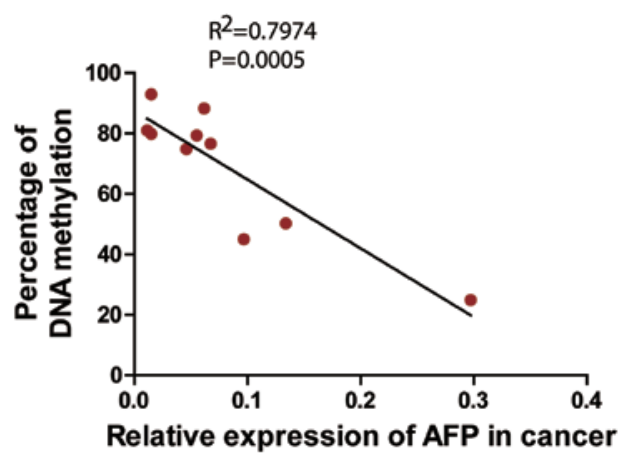

Figure 5. (A) AFP mRNA expression by reverse transcription-quantitative polymerase chain reaction in an independent cohort of 10 paired HBs and adjacent non-tumor tissues with complementary DNA $(\mathrm{P}<0.01$; paired Student's $t$-test). (B) Correlation analysis of $A F P$ mRNA expression and percentage of DNA methylation in $10 \mathrm{HB}$ primary tissues, analyzed by two-tailed Pearson's correlation test. AFP, alpha-fetoprotein; HB, hepatoblastoma; NT, non-tumor; mRNA, messenger RNA.
Hypermethylation or hypomethylation of gene promoter regions can result in their transcriptional silencing or activation (24). Although genome-wide copy number alterations in HB have been studied, little is known about genome-wide methylation changes (4). The present study is the first to explore whole-genome DNA methylation status in HB. In the present study, Infinium HumanMethylation450 BeadChip, a new platform for high-throughput DNA methylation analysis, was used (25). The cluster analysis revealed markedly less methylation in HB tissues than in normal liver tissues in the three sets of paired tissues analyzed. HB tissues are relatively low in genome-wide DNA methylation with preponderant $\mathrm{CpG}$ sites, and highly methylated genes may also have more numerous $\mathrm{CpG}$ sites (26). The present results revealed the DNA in HB tissues to be less methylated than in normal liver tissues. This may explain how the disease begins: A number of oncogenes are activated by demethylation, resulting in rapid tumor growth.

Serum $A F P$ screening for HB has been a clinical practice for a long time (27). Additionally, high mRNA levels of $A F P$ are reportedly associated with HB (27). To further understand the association between HB and AFP methylation, a methylation microarray was used in the present study to specifically detect AFP methylation status. A methylation-enriched position near the AFP transcription start site [g10778295 (TSS1500)] was identified, which was hypomethylated in HB. Pyrosequencing demonstrated the methylation level of the AFP locus cg10778295 to be lower in cancer tissues $(79 \%)$ than in normal liver tissues (91\%). Finally, RT-qPCR 
demonstrated AFP mRNA expression in $\mathrm{HB}$ to be much higher than in normal liver tissue in an independent cohort of 10 adjacent HB-non-tumor tissues pairs. The expression of $A F P$ mRNA was significantly negatively correlated with its methylation status.

In conclusion, the current study of methylation in $\mathrm{HB}$ tissues is a proof-of-principle that methylation status probably affects $\mathrm{HB}$ development and progression. $\mathrm{HB}$ is an uncommon malignant liver neoplasm of children, and its etiology, pathophysiology and molecular mechanisms are largely unknown. Further studies are required to fully understand and control this disease. Although the current study demonstrated only that high $A F P$ expression is associated with epigenetic modification, this topic warrants further investigation.

\section{Acknowledgements}

The present study received financial support from the National Natural Science Foundation of China (Beijing, China; grant nos. 81370472 and 81300517), the Shanghai City Health Bureau for Youth Scientific Fund Project (Shanghai, China; grant no. 20134y100), the Science Foundation of Shanghai (Shanghai, China; grant nos. 11JC1401300, 13ZR1451800 and 15ZR1404200) and the State Key Laboratory of Oncogenes and Related Genes, Shanghai Cancer Institute (Shanghai, China; grant no. SKLORG \#90-12-01).

\section{References}

1. Herzog CE, Andrassy RJ and Eftekhari F: Childhood cancers: Hepatoblastoma. Oncologist 5: 445-453, 2000.

2. von Schweinitz D: Hepatoblastoma: Recent developments in research and treatment. Semin Pediatr Surg 21: 21-30, 2012.

3. De Ioris M, Brugieres L, Zimmermann A, Keeling J, Brock P, Maibach R, Pritchard J, Shafford L, Zsiros J, Czaudzerna P and Perilongo G: Hepatoblastoma with a low serum alpha-fetoprotein level at diagnosis: The SIOPEL group experience. Eur J Cancer 44: 545-550, 2008.

4. Litten JB and Tomlinson GE: Liver tumors in children. Oncologist 13: 812-820, 2008.

5. Haas JE, Feusner JH and Finegold MJ: Small cell undifferentiated histology in hepatoblastoma may be unfavorable. Cancer 92: 3130-3134, 2001

6. Geiman TM and Robertson KD: Chromatin remodeling, histone modifications and DNA methylation-how does it all fit together? J Cell Biochem 87: 117-125, 2002.

7. Reik W and Walter J: Genomic imprinting: Parental influence on the genome. Nat Rev Genet 2: 21-32, 2001.

8. Chow J and Heard E: X inactivation and the complexities of silencing a sex chromosome. Curr Opin Cell Biol 21: 359-366, 2009.

9. Bibikova M, Barnes B, Tsan C, Ho V, Klotzle B, Le JM, Delano D, Zhang L, Schroth GP, Gunderson KL, et al: High density DNA methylation array with single $\mathrm{CpG}$ site resolution. Genomics 98 : 288-295, 2011.
10. Doi A, Park IH, Wen B, Murakami P, Aryee MJ, Irizarry R, Herb B, Ladd-Acosta C, Rho J, Loewer S, et al: Differential methylation of tissue- and cancer-specific $\mathrm{CpG}$ island shores distinguishes human induced pluripotent stem cells, embryonic stem cells and fibroblasts. Nat Genet 41: 1350-1353, 2009.

11. Irizarry RA, Ladd-Acosta C, Wen B, Wu Z, Montano C, Onyango P, Cui H, Gabo K, Rongione M, Webster M, et al: The human colon cancer methylome shows similar hypo- and hypermethylation at conserved tissue-specific $\mathrm{CpG}$ island shores. Nat Genet 41: 178-186, 2009.

12. Ogoshi K, Hashimoto S, Nakatani Y, Qu W, Oshima K, Tokunaga K, Sugano S, Hattori M, Morishita S and Matsushima K: Genome-wide profiling of DNA methylation in human cancer cells. Genomics 98: 280-287, 2011

13. Revill K, Wang T, Lachenmayer A, Kojima K, Harrington A, Li J, Hoshida Y, Llovet JM and Powers S: Genome-wide methylation analysis and epigenetic unmasking identify tumor suppressor genes in hepatocellular carcinoma. Gastroenterology 145: 1424-1435.e1-e25, 2013.

14. Sandoval J, Heyn H, Moran S, Serra-Musach J, Pujana MA, Bibikova $\mathrm{M}$ and Esteller M: Validation of a DNA methylation microarray for $450,000 \mathrm{CpG}$ sites in the human genome. Epigenetics 6: 692-702, 2011.

15. Dennis G Jr, Sherman BT, Hosack DA, Yang J, Gao W, Lane HC and Lempicki RA: DAVID: Database for annotation, visualization, and integrated discovery. Genome Biol 4: P3, 2003.

16. Johnson MR, Wang KS, Smith JB, Heslin MJ and Diasio RB: Quantitation of dihydropyrimidine dehydrogenase expression by real-time reverse transcription polymerase chain reaction. Anal Biochem 278: 175-184, 2000.

17. Perek B, Malinska A, Stefaniak S, Ostalska-Nowicka D, Misterski M, Zabel M, Suri A and Nowicki M: Predictive factors of late venous aortocoronary graft failure: Ultrastructural studies. PLoS One 8: e706288, 2013.

18. Kingston JE, Herbert A, Draper GJ and Mann JR: Association between hepatoblastoma and polyposis coli. Arch Dis Child 58: 959-962, 1983.

19. Ross JA: Hepatoblastoma and birth weight: Too little, too big, or just right? J Pediatr 130: 516-517, 1997.

20. Mamlok V, Nichols M, Lockhart L and Mamlok R: Trisomy-18 and hepatoblastoma. Am J Med Genet 33: 125-126, 1989.

21. Takayasu H, Horie H, Hiyama E, Matsunaga T, Hayashi Y, Watanabe Y, Suita S, Kaneko M, Sasaki F, Hashizume K, et al: Frequent deletions and mutations of the beta-catenin gene are associated with overexpression of cyclin D1 and fibronectin and poorly differentiated histology in childhood hepatoblastoma. Clin Cancer Res 7: 901-908, 2001.

22. Jia D, Dong R, Jing Y, Xu D, Wang Q, Chen L, Li Q, Huang Y, Zhang Y, Zhang Z, et al: Exome sequencing of hepatoblastoma reveals novel mutations and cancer genes in the Wnt pathway and ubiquitin ligase complex. Hepatology 60: 1686-1696, 2014.

23. Dong R, Jia D, Xue P, Cui X, Li K, Zheng S, He X and Dong K: Genome-wide analysis of long noncoding RNA (lncRNA) expression in hepatoblastoma tissues. PLoS One 9: e85599, 2014.

24. Baylin SB and Ohm JE: Epigenetic gene silencing in cancer-a mechanism for early oncogenic pathway addiction? Nat Rev Cancer 6: 107-116, 2006.

25. Morris TJ, Butcher LM, Feber A, Teschendorff AE, Chakravarthy AR, Wojdacz TK and Beck S: ChAMP: 450k chip analysis methylation pipeline. Bioinformatics 30: 428-430, 2014.

26. Tomlinson GE and Kappler R: Genetics and epigenetics of hepatoblastoma. Pediatr Blood Cancer 59: 785-792, 2012.

27. Clericuzio CL, Chen E, McNeil DE, O'Connor T, Zackai EH, Medne L, Tomlinson G and DeBaun M: Serum alpha-fetoprotein screening for hepatoblastoma in children with Beckwith-Wiedemann syndrome or isolated hemihyperplasia. J Pediatr 143: 270-272, 2003. 